\title{
CHARMONIUM DECAY PHYSICS
}

\author{
Y.F. Gu and S.F. Tuan ${ }^{\mathrm{b}}$ \\ ${ }^{a}$ Institute of High Energy Physics, Beijing, China \\ ${ }^{\mathrm{b}}$ University of Hawaii at Manoa, Honolulu, USA
}

UH-511-944-99

BIHEP-EP1-99-04

October 1999

Recent experimental results on the decays of charmonium, together with related physics issues, are reviewed. Some future prospects are described.

\section{INTRODUCTION}

The dramatic discovery of charmonium, the $J / \psi$ and its radial excitation $\psi(2 S)$, launched the modern era of particle physics. After a hiatus of about one decade in the 1980's following a period of several-years of intense experimental activity, charmonium physics has emerged again as one of the most exciting areas of experimental high energy physics. A wealth of new data in the last few years has changed greatly the face of this area.

As the "hydrogen atom of strong interaction physics", charmonium states have been studied in many experiments, which basically use three techniques: formation and subsequent cascade decays from $e^{+} e^{-}$annihilations, two virtual photon interactions from high energy $e^{+} e^{-}$collisions and formation from $\bar{p} p$ collisions. At present the Beijing Spectrometer (BES) is the only experiment at the $e^{+} e^{-}$collider (BEPC) to study charmonium physics around the $c \bar{c}$ threshold in $e^{+} e^{-}$annihilations. The detectors at CESR and LEP, such as CLEO, DELPHI, L3, and OPAL, are performing experiments on two photon physics. The Fermilab experiment E760 and its upgraded experiment E835 are studying the direct formation of $c \bar{c}$ states in $\bar{p} p$ annihilations at the Fermilab Antiproton Accumulator Ring. Precision measurements of the $c \bar{c}$ system (masses, widths, decay rates, etc.) are important inputs to test the limit of PQCD and the order of magnitude of relativistic and radiative corrections.

Recent theoretical developments in effective field theories such as nonrelativistic QCD and heavy quark effective theory, lattice gauge theory, and light front quantization suggest that it should be possible to place the theory of charmonium on a rigorous foundation that is derived directly from QCD.

\section{REVIEW OF CHARMONIUM DATA}

In this section we will review the experimental data of charmonium states below $D \bar{D}$ threshold. The subjects discussed are the mass, width, and other parameters of $1^{3} S_{1}, 2^{3} S_{1},{ }^{3} P_{0,1,2}, 1^{1} S_{0}$, $2^{1} S_{0}$, and $1^{1} P_{1}$ resonances. We emphasize on the results obtained since 1990 . 


\section{1. $1^{3} S_{1}: J / \psi$}

A high precision measurement was performed by BES[1] on leptonic branching fractions from a comparison of the exclusive and inclusive processes: $\psi(2 S) \rightarrow \pi^{+} \pi^{-} J / \psi$, with $J / \psi \rightarrow l^{+} l^{-}$ and $J / \psi \rightarrow$ anything, which is luminosity independent and almost free of QED backgrounds. The BES[1] obtained values for $B\left(J / \psi \rightarrow e^{+} e^{-}\right)=5.90 \pm 0.05 \pm 0.10 \%$ and $B\left(J / \psi \rightarrow \mu^{+} \mu^{-}\right)=$ $5.84 \pm 0.06 \pm 0.10 \%$. Including BES data, the new world average will have an error less than 1.5 \%, which is about a factor of two improvement over the 1998 PDG value[2].

\section{2. $2^{3} S_{1}: \psi(2 S)$}

E760 has reported the first direct measurement of the total width of the $\psi(2 S)[3], \Gamma=$ $306 \pm 36 \pm 16 \mathrm{keV}$. Compared to the value derived from a review of all previous data in 1992 《], $\Gamma=243 \pm 43 \mathrm{keV}$, the central value of E760 is larger. E760 performed new measurements on the branching fractions of $\psi(2 S)$ decays to $J / \psi \pi^{+} \pi^{-}, J / \psi \pi^{0} \pi^{0}$, and $J / \psi \eta$ and claimed that they are able to make measurements of $B\left(J / \psi \pi^{+} \pi^{-}\right)$and $B\left(J / \psi \pi^{0} \pi^{0}\right)$ with errors comparable to the world average [5]. However, as has been pointed out by $\mathrm{Gu}$ and $\mathrm{Li}[6]$, there is logical inconsistency in handling of the computational procedure in Ref. [5]. As also pointed out by $\mathrm{Gu}$ and $\mathrm{Li}[6]$, the ratio of $B\left(J / \psi \pi^{+} \pi^{-}\right) / B\left(\mu^{+} \mu^{-}\right)$measured by E672/E706[7] as equal to $30.2 \pm 7.1 \pm 6.8$, was mistaken for $B\left(J / \psi \pi^{+} \pi^{-}\right) / B\left(J / \psi \mu^{+} \mu^{-}\right)$in PDG 1998[2]. They thus suggested that we not use the 1998 PDG fit values of branching fractions for the $\psi(2 S)$ decays to $J / \psi$ plus anything [6]. PDG will provide new fit values for $B(J / \psi+$ anything $), B(J / \psi+$ neutrals $), B\left(J / \psi \pi^{+} \pi^{-}\right)$, $B\left(J / \psi \pi^{0} \pi^{0}\right), B(J / \psi \eta), B\left(\gamma \chi_{c 0}\right), B\left(\gamma \chi_{c 1}\right)$, and $B\left(\gamma \chi_{c 2}\right)$ in the next edition by removing the E760 data and correcting the above mistake [B].

Using the world's largest data sample of $\psi(2 S)$, BES has measured $\psi(2 S)$ branching fractions for a large number of hadronic final states - many for the first time[9-12]. The results for 2-body (light) meson final states will be discussed in the next section in the context of hadronic decay puzzle.

\section{3. ${ }^{3} P_{0,1,2}: \chi_{c 0}, \chi_{c 1}, \chi_{c 2}$}

The large sample of $\psi(2 S)$ decays at BES permits the study of $\chi_{c J}$ decays with unprecedented precision. Using many decay modes of the $\chi_{c 0}$, BES has determined $M\left(\chi_{c 0}\right)=3414.1 \pm 0.6 \pm 0.8$ $\mathrm{MeV}$ [13]. The precision of this measurement represents a substantial improvement over the existing PDG value of $3417.3 \pm 2.8[2]$. BES also determined the $\chi_{c 0}$ total width 14], $\Gamma\left(\chi_{c 0}\right)=$ $14.3 \pm 2.0 \pm 3.0 \mathrm{MeV}$, by selecting a $\pi^{+} \pi^{-}$event sample, using the precisely measured total width of the $\chi_{c 2}$ [2] to determine the detector resolution and a $\mathrm{MC}$ simulation to determine how the resolution changes from $M\left(\chi_{c 2}\right)$ to $M\left(\chi_{c 0}\right)$. Compared with the only existing result of Crystal Ball[15], $\Gamma\left(\chi_{c 0}\right)=13.5 \pm 3.3 \pm 4.2 \mathrm{MeV}$, which is actually a combination of two measurements with large errors and of only marginal consistency (within $2.2 \sigma$ ), the uncertainty is now reduced from $40 \%$ to $25 \%$.

$P$-wave charmonium states are directly accessible in $\bar{p} p$ annihilations. Precision measurements of the masses and the total widths of the $\chi_{c 1}$ and $\chi_{c 2}$ resonances were performed by E760 using the line shape method a few years ago[16]. The results are given in Table 1. These new values of the masses agree well with earlier measurements [2]; the errors are reduced by more than a factor of two. The width of the $\chi_{c 1}$ has been measured for the first time; the uncertainty on the $\chi_{c 2}$ width has been reduced from about $40 \%$ to about $10 \%$.

While there are only upper limits on $\gamma \gamma$ partial widths for the $\chi_{c 0}$ resonance exist so far [14, 15], there are a number of measurements for the $\chi_{c 2}$ made by L3 18, 19], E835 20], OPAL[21], CLEO 2], 22], E760[2], and TPC[2] since 1990. For $\chi_{c 0}$, the limits are $\Gamma_{\gamma \gamma}<6.2 \mathrm{keV}$ reported by CLEO and $<5.5 \mathrm{keV}$ reported recently by L3 (both 95\% C.L.); the only branching fraction measured by Crystal Ball was never actually published[23]. The results for $\chi_{c 2}$ are summarized in Table 2 . 
Table 1

E760 measurements of $\chi_{c 1}$ and $\chi_{c 2}$ parameters

\begin{tabular}{|c|c|c|}
\hline${ }^{3} P_{J}$ state & Mass $(\mathrm{MeV})$ & Width $(\mathrm{MeV})$ \\
\hline$\chi_{c 1}$ & $3510.53 \pm 0.04 \pm 0.12$ & $0.88 \pm 0.11 \pm 0.08$ \\
\hline$\chi_{c 2}$ & $3556.12 \pm 0.07 \pm 0.12$ & $1.98 \pm 0.17 \pm 0.07$ \\
\hline
\end{tabular}

Among these data, the central value of $\Gamma_{\gamma \gamma}$ for $\chi_{c 2}$ differs significantly, and measurements at the $e^{+} e^{-}$colliders (LEP,CESR,PEP) seem all larger than the E760/E835 results obtained in $\bar{p} p$ annihilations. New measurements are still required. A reduction in the discrepancy found between the $\chi_{c 2}$ data will be of fundamental importance to guide the extraction of theoretical parameters from the data.

Table 2

$\gamma \gamma$ partial width for $\chi_{c 2}$

\begin{tabular}{|l|c|}
\hline Experiment & $\Gamma_{\gamma \gamma}\left(\chi_{c 2}\right)(\mathrm{keV})$ \\
\hline L3 $[18]$ & $<1.4(95 \%$ C.L $)$ \\
\hline E835 $[20]$ & $0.311 \pm 0.041 \pm 0.031($ prelim $)$ \\
\hline L3[19] & $1.02 \pm 0.40 \pm 0.15 \pm 0.09$ \\
\hline OPAL[21] & $1.76 \pm 0.47 \pm 0.37 \pm 0.15$ \\
\hline CLEO $[22]$ & $0.7 \pm 0.2 \pm 0.1 \pm 0.2$ \\
\hline CLEO[2] & $1.08 \pm 0.30 \pm 0.26$ \\
\hline E760[2] & $0.321 \pm 0.078 \pm 0.054$ \\
\hline TPC $[2]$ & $3.4 \pm 1.7 \pm 0.9$ \\
\hline
\end{tabular}

BES performed the first measurement of the branching fraction and the partial width for $\chi_{c 0} \rightarrow \bar{p} p$ 14. After publication of the BES results for all $\chi_{c J} \rightarrow \bar{p} p$ branching fractions, E835 also reported its first measurements on $\chi_{c 0}$ and new results on $\chi_{c 1}$ and $\chi_{c 2}$ [20]. The results are compared in Table 3 with BES measurements. One notes that E760/E835 results for all $B\left(\chi_{c J} \rightarrow \bar{p} p\right)$ are systematically higher (tantalizingly large! - C. Quigg 24] with respect to BES results, though both have large errors. It has been pointed out [24] that in both experiments the $B_{\bar{p} p}$ and $\Gamma_{\bar{p} p}$ are derived from the product of branching fractions $B_{\text {in }} \times B_{\text {out }}$ and from $B\left(\psi(2 S) \rightarrow \gamma \chi_{c J}\right)$ and $B\left(\chi_{c J} \rightarrow \gamma J / \psi\right)$ respectively. New measurements of these two branching fractions would be desirable to exclude one possible origin of such inconsistency.

BES studied many other hadronic decays of $P$-wave charmonium states, and determined altogether more than 30 branching fractions for $\chi_{c 0}, \chi_{c 1}$ and $\chi_{c 2}$ 12, 13, using the PDG values for $B\left(\psi(2 S) \rightarrow \gamma \chi_{c J}\right)[2]$. Among them 15 were measured for the first time.

2.4. $1^{1} S_{0}: \eta_{c}$

In spite of a number of measurements on the mass of $\eta_{c}$, the value remains ambiguous in PDG 1998 edition[2]. The PDG average there is based on a fit to 7 measurements with poor internal consistency and the confidence level is only 0.001. The measurement of E760 25] disagrees with the value of DM2 26] by almost $4 \sigma$ and is almost $10 \mathrm{MeV}$ different from 1994 PDG average. The change will cause a shift in the value of the hyperfine splitting for the $S$-wave charmonium states which, in turn, are important in understanding the spin-spin forces.

The value of $M\left(\eta_{c}\right)$ determined recently by BES using several decay modes of the $\eta_{c}[13$ is in 
Table 3

Comparison of BES and E760/E835 results for $\chi_{c J}$ decays $\mathrm{B}(\bar{p} p) \times 10^{4}$ (left) and $\Gamma(\bar{p} p)$ in $\mathrm{keV}$ (right).

\begin{tabular}{|c|c|c|c|c|}
\hline${ }^{3} P_{J}$ & BES & E760/E835 & BES & E760/E835 \\
\hline$\chi_{c 0}$ & $1.59 \pm 0.43 \pm 0.53$ & $4.82_{-0.81-1.12}^{+0.97+2.08}$ & $2.3 \pm 1.1$ & $8.0_{-1.6-1.9}^{+1.9+3.5}$ \\
\hline$\chi_{c 1}$ & $0.42 \pm 0.22 \pm 0.28$ & $0.78 \pm 0.10 \pm 0.11$ & $0.037 \pm 0.032$ & $0.069 \pm 0.009$ \\
& & $(\mathrm{E} 835)$ & & $\pm 0.010(\mathrm{E} 835)$ \\
& & $0.86 \pm 0.12(\mathrm{E} 760)$ & & $0.076 \pm 0.010$ \\
& & & & $\pm 0.005(\mathrm{E} 760)$ \\
\hline$\chi_{c 2}$ & $0.58 \pm 0.31 \pm 0.32$ & $0.91 \pm 0.08 \pm 0.14$ & $0.116 \pm 0.090$ & $0.180 \pm 0.016$ \\
& & $(\mathrm{E} 835)$ & & $\pm 0.026(\mathrm{E} 835)$ \\
& & $1.00 \pm 0.11(\mathrm{E} 760)$ & & $0.197 \pm 0.018$ \\
& & & & $\pm 0.016(E 760)$ \\
\hline
\end{tabular}

excellent agreement with DM2 data and is $2.4 \sigma$ below the E760 result. More recently, L3 has also reported their measurement on the $\eta_{c}$ mass 18], which agrees well both with BES and DM2. A comparison of recent results is shown in Table 4 .

Table 4

Recent data on $\eta_{c}$ mass

\begin{tabular}{|l|l|}
\hline Experiment & Mass of $\eta_{c}(\mathrm{MeV})$ \\
\hline L3[18] & $2974 \pm 18$ \\
\hline BES[13] & $2975.8 \pm 3.9 \pm 1.2$ \\
\hline E760[25] & $2988.3_{-3.1}^{+3.3}$ \\
\hline DM2[26] & $2974.4 \pm 1.9$ \\
\hline MARK3 [27] & $2969 \pm 4 \pm 4$ \\
\hline
\end{tabular}

New measurements on $\eta_{c}$ width was made by E760[25], $\Gamma=23.9_{-7.1}^{+12.6} \mathrm{MeV}$, and improved by E835 afterwards 20] with $\Gamma=17.8_{-6.9}^{+7.2} \mathrm{MeV}$ (preliminary). The results are still larger than previous measurements [2] where for instance the Crystal Ball value in 1986 is listed as $11.5 \pm 4.5$ $\mathrm{MeV}$, and the errors remain large. The $\gamma \gamma$ partial width of $\eta_{c}$ was measured by a number of experiments, including L3[18], E760/E835[2]20], ARGUS[2] and CLEO[2]22]. Unfortunately, the data are not of sufficient precision to differentiate between theories.

\section{5. $2^{1} S_{0}: \eta_{c}(2 S)$}

After observation of a candidate of this state by Crystal Ball experiment at $3594 \mathrm{MeV}$ [28], it has been searched for in E835 20], BES 13], DELPHI 29], and L3 18] recently. No evidence is found in the mass region around $3594 \mathrm{MeV}$ by any of the subsequent experiments. This appears to challenge the theoretical analysis of Barnes, Browder, and Tuan [30] based on the relationship that hadronic branching fractions of $\eta_{c}$ and $\eta_{c}(2 S)$ to the same exclusive final state channel could be equal[31] and a nonrelativistic quark potential model calculation 32] that $\Gamma\left(\eta_{c}(2 S) \rightarrow \gamma \gamma\right)=3.7 \mathrm{keV}$. The L3[18] upper limit on $\Gamma_{\gamma \gamma}\left(\eta_{c}(2 S)\right)<2.0 \mathrm{keV}$ (95\% C.L.) is not yet a severe constraint since the model calculation of this partial width might only be good to a factor of 2 to 332. Search for $\eta_{c}(2 S)$ in the two photon process at CESR, which has already delivered more than $11 \mathrm{fb}^{-1}$ of integrated luminosity to CLEO, remains a valuable goal. We 
must caution however that though the important observation that $\gamma \gamma$ widths are not strongly suppressed with radial excitation in any of the $q \bar{q}$ systems considered[30], to date no radial excitations have been identified in $\gamma \gamma$ collisions, so for the present this width calculation should be taken as a theoretical estimate in a regime in which theory has not been tested.

\section{6. ${ }^{1} P_{1}: h_{c}(1 P)$}

E760 announced the discovery of this state at 3526.14 MeV[33]. In a subsequent search for hidden charm states in $\pi^{-}$- and $p-L i$ interactions, E705 reported the observation of a $J / \psi \pi^{+} \pi^{-}$ signal at $3.836 \mathrm{GeV}$ (possible ${ }^{3} D_{2}$ state) and a $J / \psi \pi^{0}$ enhancement at $3.527 \mathrm{GeV}$ (possibly the ${ }^{1} P_{1}$ state) [34]. However, E672/E706 has questioned the strong structure at $3.836 \mathrm{GeV}$ [7]. It was also questioned by Barnes, Browder, and Tuan 35] whether E705 could have 'confirmed' E760's discovery of ${ }^{1} P_{1}$ state. E835 will continue this work and look further with more data in the near future.

\section{CHARMONIUM HADRONIC DECAY PUZZLE}

This celebrated " $\rho-\pi$ " puzzle with dramatic suppression of $\psi(2 S) \rightarrow V P[36,37$ and $V T[9]$, but apparent non suppression of $\psi(2 S) \rightarrow A P, V S$ as well as isospin violating modes $\omega \pi^{0}, \rho \eta^{0}$ (with branching ratios in accord with PQCD "14\%" rule), has been mostly summarized in 38]. We note in particular that BES has concluded that $\psi(2 S) \rightarrow \omega \pi^{0}$ is larger than (strong) isospin conserving, $\mathrm{SU}(3)$-allowed, $\psi(2 S) \rightarrow \rho \pi$ decay while large isospin violations are seen between branching fractions for charged and neutral $\psi(2 S) \rightarrow K^{*} \bar{K}$ decays.

The failure of most theoretical models up to 1990 have been summarized in Table 5, while those proposed in recent years have been summarized in [39]. Actually the model of Li-Bugg-Zou (LBZ for short) [40], though fortuitous 39], cannot be ruled out. Based on final state interaction FSI, Suzuki 41] nevertheless pointed out that their numerical computation picks two completely arbitrary intermediate states in estimating the FSI effects. One can in fact get almost any number by selecting intermediate states of one's choice. Unfortunately the intermediate states picked by LBZ are in fact heavily dominated by other intermediate states. Hence LBZ model does not answer the question of which specific FSI is responsible for the puzzle.

The most recent model of Gérard and Weyers 42 has the following problems. (a) The BES data 11] that for $\psi(2 S) \rightarrow A P$ with $K_{1}(1270) \bar{K}$ (large) and $K_{1}(1400) \bar{K}$ (small), cannot be clearly understood in the model. (b) The universality assumption for three- gluon hadronization of $\psi(1 S)$ remains doubtful[43]. For instance the three gluons from $\psi(1 S)$ must certainly hadronizes to say $V P$ and $V T$ final states in different ways. Can the phase really be the same? (c) The model emphasizes on $\psi(2 S) \rightarrow A P, A S$ final states to leading order. Hence unsuppressed $\psi(2 S) \rightarrow \phi f_{0}(980)$ [44,12] a $V S$ mode, and $\psi(2 S) \rightarrow K^{* 0} \bar{K}^{* 0}$ a $V V$ mode 12] would appear to be at variance with the model. There is a need for further concerted effort on both theoretical and experimental side to provide a solution to the $J / \psi / \psi(2 S) \rightarrow \rho \pi$ puzzle.

The $\rho-\pi$ puzzle motivated the important discovery of long-distance (large phase) FSI physics 45 47 from $J / \psi \rightarrow V P, P P, B \bar{B}$ data. Its resolution remains very important. For instance Suzuki noted 45 that in $B$-meson decays knowledge of much higher precision will be needed for FSI phases above the inelastic thresholds, a nearly impossible task for theoretical computation/extraction from scattering data. Parameters of fundamental interactions can then only be extracted from data free from FSI (a severe limitation?). Also [47] stressed that FSI in nonleptonic $B$-decay has been an important unsolved issue in direct search for $C P$ violations.

Rosner did significant damage control [48,49] for the future of $B$-Factory physics. He introduced (i) universal FSI as consequence that $\gamma$ and $3 \mathrm{~g}$ amplitudes for $J / \psi$ are out of phase 
Table 5

Theoretical models up to 1990 and related experimental results.

\begin{tabular}{|c|c|c|}
\hline Model & Predictions & Experimental results \\
\hline $\begin{array}{l}\text { Brodsky-Lepage-Tuan } \\
(1987) ; \\
\text { Hou-Soni } \\
(1983)\end{array}$ & $\begin{array}{l}\text { Hadron helicity conserved: } \\
\text { - } \psi(2 S) \rightarrow \text { VP modes } \\
\text { suppressed; } \\
\text { - } \psi(2 S) \rightarrow \text { VT modes } \\
\text { not suppressed. } \\
J / \psi \text {-glueball mixing: } \\
\text { - } J / \psi \text { shape distorted; } \\
\text {-Search in } \psi(2 S) \rightarrow \pi^{+} \pi^{-} O \\
\text { - } J / \psi \rightarrow \phi f_{0} \text { enhanced. }\end{array}$ & $\begin{array}{l}-\psi(2 S) \rightarrow \omega \pi^{0} \\
\quad \text { not suppressed; } \\
-\psi(2 S) \rightarrow \mathrm{VT} \\
\quad \text { suppressed. } \\
\text {-Not seen; limits set; } \\
\text {-Not seen; limits set. } \\
-\psi(2 S) \rightarrow \phi f_{0} \\
\text { not suppressed. }\end{array}$ \\
\hline $\begin{array}{l}\text { Chaichian-Tornqvist } \\
(1989)\end{array}$ & $\begin{array}{l}\text { Energy dependent } \\
\text { exponetial form factor: } \\
-\psi(2 S) \text { 2-body meson modes } \\
\text { suppressed; } \\
\text {-B }(\psi(2 S) \rightarrow \rho \pi)=7 \times 10^{-5}\end{array}$ & $\begin{array}{l}-b_{1} \pi \text { and } \phi f_{0} \\
\text { not suppressed; } \\
-\mathrm{B}(\psi(2 S) \rightarrow \rho \pi) \\
<2.8 \times 10^{-5}\end{array}$ \\
\hline $\begin{array}{l}\text { Pinsky } \\
(1990)\end{array}$ & $\begin{array}{l}\psi(2 S) \rightarrow \mathrm{VP} \text { are hindered } \\
\mathrm{M} 1 \text { transitions: } \\
-\mathrm{B}\left(\psi(2 S) \rightarrow \gamma \eta^{\prime}\right)=9 \times 10^{-6} \\
-\mathrm{B}(\psi(2 S) \rightarrow \rho \pi)=4 \times 10^{-5} \\
-\psi(2 S) \rightarrow \omega f_{2} \\
\text { not suppressed. }\end{array}$ & $\begin{array}{l}-\mathrm{B}\left(\psi(2 S) \rightarrow \gamma \eta^{\prime}\right) \\
=150 \times 10^{-6} ; \\
-\mathrm{B}(\psi(2 S) \rightarrow \rho \pi) \\
<2.8 \times 10^{-5} ; \\
-\psi(2 S) \rightarrow \omega f_{2} \\
\text { suppressed. }\end{array}$ \\
\hline
\end{tabular}

$(\simeq \pi / 2)$ with each other. (ii) Connection is made with charmonium where strong phase shifts in $B \rightarrow P P$ arise as result of strong absorptive effects in rescattering of $c \bar{c} \rightarrow$ light quarks 448. (iii) Predicts (c.f. Table VI of [48]) direct $C P$ asymmetries in $B^{0}\left(\bar{B}^{0}\right) \rightarrow K^{+} \pi^{-}\left(K^{-} \pi^{+}\right)$, $B^{ \pm} \rightarrow K^{ \pm} \pi^{0}$ maximally $\sim 0.34$. (iv) Emphasized decays of neutral $B$ mesons to $C P$ eigenstates such as $J / \psi K_{S}^{0}$ and $\pi \pi$ can directly probe CKM phases, since their interpretation is immune from strong FSI. Hence recent measurement of $\sin 2 \beta$ [50], a $C P$ violating parameter, remains valid. (v) Suzuki [51] has continued this favorable ambiance with a very recent paper on testing direct $C P$ violation of standard model without knowing strong phases.

Since large $C P$ asymmetry would require large FSI 43, CLEO III with a single ring and a well tried detector (suitably upgraded) could be decisive in the study of $C P$ asymmetries for $B^{0}\left(\bar{B}^{0}\right) \rightarrow K^{+} \pi^{-}\left(K^{-} \pi^{+}\right), B^{ \pm} \rightarrow K^{ \pm} \pi^{0}$ before year end.

Emphasis on $J / \psi / \psi(2 S)$ physics should not detract us from the significant physics to be done in the open charm domain. For instance, the $D$ can be fully reconstructed in $\psi^{\prime \prime}(3.772) \rightarrow D \bar{D}$, while $\bar{D} \rightarrow \mu+\nu$ can be deployed to measure $f_{D}$. Currently $f_{D}<290 \mathrm{MeV}$ and $f_{D_{s}}=250 \mathrm{MeV}$, while $S U(3)$ breaking suggests $f_{D_{s}} / f_{D}=1.1-1.25$, so $f_{D} \sim 200-220 \mathrm{MeV}$ (an attainable experimental goal). Grinstein 52] says that up to $5 \%$, we have

$$
f_{B_{s}} / f_{B} \simeq f_{D_{s}} / f_{D}, \text { and }\left[\Delta M_{s} / \Delta M_{d}\right]^{1 / 2} \simeq \frac{\left|V_{t s}\right|}{\left|V_{t d}\right|}\left(f_{B_{s}} / f_{B}\right)
$$


where $\Delta M_{s}$ and $\Delta M_{d}$ are $B \bar{B}$ splittings in strange/non strange $B$ respectively. So we are again back to fundamentals of measuring CKM matrix elements! Finally with the advent of a Tau-Charm Factory, we must not forget about exploration of molecular charmonium states as discussed recently[53].

\section{FUTURE PROSPECTS}

E835 experiment will continue to take data during 1999-2000 period, with $20 p b^{-1}$ accumulation of $\chi_{c 0}, 100 p b^{-1}$ of $\eta_{c}(2 S)$, and $200 p b^{-1}$ of $\psi\left({ }^{1} P_{1}\right)$ anticipated respectively. At the forthcoming run at BES, accumulation of $5 \times 10^{7} \mathrm{~J} / \psi$ are expected, while there is a proposal for $2 \times 10^{7} \psi(2 S)$ run. It is to be hoped that there will also be a run at $\psi^{\prime \prime}(3.772)$ for open charm study. Then there are the $B$-Factories Babar/PEP II, Belle/KEK-B and CLEO III. Many in the high

energy physics community feel that charm spectroscopy both below and above $D \bar{D}$ threshold is fascinating and badly needs a new high statistics facility. A Tau-Charm Factory with luminosity about two orders of magnitude higher than the BEPC would fill this need.

\section{REFERENCES}

1. BES Collaboration, J.Z. Bai et al., Phys. Rev. D 58 (1998) 092006.

2. Particle Data Group, C. Caso et al., Eur. Phys. J. C 3 (1998) 1.

3. E760 Collaboration, T.A. Armstrong et al., Phys. Rev. D 47 (1993) 772.

4. Particle Data Group, K. Hikasa et al., Phys. Rev. D 45 (1992), 1 June, Part II.

5. E760 Collaboration, T.A. Armstrong et al., Phys. Rev. D 55 (1997) 1153.

6. Y.F. Gu and X.H. Li, Phys. Lett. B 449 (1999) 361.

7. E672/E706 Collaboration, A. Gribushin et al., Phys. Rev. D 53 (1996) 4723.

8. C. Amsler (PDG), private communication (1998).

9. BES Collaboration, J.Z. Bai et al., Phys. Rev. Lett. 81 (1998) 5080.

10. BES Collaboration, J.Z. Bai et al., Phys. Rev. D 58 (1998) 097101.

11. BES Collaboration, J.Z. Bai et al., Phys. Rev. Lett. 83 (1999) 1918.

12. F. Liu (BES Collaboration), these Proceedings.

13. BES Collaboration, J.Z. Bai et al., Phys. Rev. D 60 (1999) 072001.

14. BES Collaboration, J.Z. Bai et al., Phys. Rev. Lett. 81 (1998) 3091.

15. Crystal Ball Collaboration, J. Gaiser et al., Phys. Rev D 34 (1986) 711.

16. E760 Collaboration, T.A. Armstrong et al., Nucl. Phys. B 373 (1992) 35; Phys. Rev. Lett. 68 (1992) 1468.

17. CLEO Collaboration, W.-Y. Chen et al., Phys. Lett. B 243 (1990) 169.

18. L3 Collaboration, M. Acciarri et al., CERN-EP/99-072, May 21, 1999, submitted to Phys. Lett. B.

19. L3 Collaboration, M. Acciarri et al., Phys. Lett. B 453 (1999) 73.

20. N. Pastrone (E835 Collaboration), Hadron Spectroscopy, Frascati, March 8-12, 1999.

21. OPAL Collaboration, K. Ackerstaff et al., Phys. Lett. B 439 (1998) 197.

22. V. Savinov and R. Fulton (CLEO Collaboration), Proc. X Intern. Workshop on PhotonPhoton Collisions, p. 203 (ed. D.J. Miller et al., Sheffield, England, 1995).

23. R. Lee (Crystal Ball Collaboration), Ph.D thesis, SLAC 282 (1985), unpublished.

24. C. Quigg, Fermilab-conf-98/390-T(1998).

25. E760 Collaboration, T.A. Armstrong et al., Phys. Rev. D 52 (1995) 4839.

26. DM2 Collaboration, D. Bisello et al., Nucl. Phys. B 351 (1991) 1.

27. MARK3 Collaboration, Z. Bai et al., Phys. Rev. Lett. 65 (1990) 1309.

28. Crystal Ball Collaboration, C. Edwards et al., Phys. Rev. Lett. 48 (1982) 70. 
29. DELPHI Collaboration, P. Abreu et al., Phys. Lett. B 441 (1998) 479.

30. T. Barnes, T.E. Browder, and S.F. Tuan, Phys. Lett. B 385 (1996) 391.

31. K.T. Chao, Y.F. Gu, and S.F. Tuan, Commun. Theor. Phys. 25 (1996) 471.

32. E.S. Ackleh and T. Barnes, Phys. Rev. D 45 (1992) 232; C.R. Münz, Bonn University Report TK-96-01 obtains/summarizes other predictions between a factor of two to three smaller than Ackleh/Barnes.

33. E760 Collaboration, T.A. Armstrong et al., Phys. Rev. Lett. 69 (1992) 2337.

34. E705 Collaboration, L. Antoniazzi et al., Phys. Rev. D 50 (1994) 4258.

35. T. Barnes, T.E. Browder, and S.F. Tuan, UH-511-868-97 (1997).

36. M.E.B. Franklin et al., Phys. Rev. Lett. 51 (1983) 963.

37. BES Collaboration, J.Z. Bai et al., Phys. Rev. D 54 (1996) 1221.

38. F.A. Harris (BES Collaboration), hep-ex/9903036 v2 9 Jul 1999; K.T. Chao, Lepton-Photon Symposium '95, p. 106 [World Scientific Publishing (1996)].

39. S.F. Tuan, hep-ph/9903332 v3 24 Mar 1999, Commun. Theor. Phys. (in press); Y.F. Gu and S.F. Tuan, Mod. Phys. Lett. A 10 (1995) 615.

40. X.-Q. Li, D.V. Bugg, and B.-S. Zou, Phys. Rev. D 55 (1997) 1421.

41. M. Suzuki, private communication.

42. J.-M. Gérard and J. Weyers, hep-ph/9906357 14 Jun 1999.

43. J.L. Rosner, private communication.

44. Y.F. Gu (BES Collaboration), Proc. of DPF '96 (Minneapolis), Vol. 2, p.986 [World Scientific Publishing (1998)].

45. M. Suzuki, Phys. Rev. D 57 (1998) 5717.

46. G. López Castro et al., hep-ph/9902300 10 Feb 1999; AIP Conference Proceedings 342 (1995) 441.

47. M. Suzuki, Phys. Rev. D 60 (1999) 051501.

48. J.L. Rosner, hep-ph/9903543 31 Mar 1999, Phys. Rev. D (in press).

49. J.L. Rosner, hep-ph/9905366 17 May 1999.

50. G. Bauer (representing the CDF Collaboration), hep-ex/9908055 19 Aug 1999.

51. M. Suzuki, hep-ph/9908420 19 Aug 1999.

52. B. Grinstein, Phys. Rev. Lett. 71 (1993) 3067; C. Glenn Boyd and B. Grinstein, Nucl. Phys. B 442 (1995) 205, ibid Nucl. Phys. B 451 (1995) 177.

53. S.F. Tuan, hep-ph/9903342 12 Mar 1999, submitted to Phys. Lett. B. 